\title{
Transitional flow in a Rushton turbine stirred tank
}

\author{
Yulong Zhang, Zhengming Gao, Zhipeng Li \\ Beijing Advanced Innovation Center for Soft Matter Science and Engineering, Beijing University of Chemical Technology, Beijing 100029, China \\ State Key Laboratory of Chemical Resource Engineering, School of Chemical Engineering, Beijing University of Chemical Technology, Beijing 100029, China \\ J.J. Derksen \\ School of Engineering, University of Aberdeen, Aberdeen AB24 3UE, UK \\ Correspondence concerning this article should be addressed to Zhipeng Li at lizp@mail.buct.edu.cn or J.J. Derksen at jderksen@abdn.ac.uk \\ AIChE Journal - Accepted - May 2017
}

\begin{abstract}
The way in which the single phase flow of Newtonian liquids in the vicinity of the impeller in a Rushton turbine stirred tank goes through a laminar - turbulent transition has been studied in detail experimentally (with Particle Image Velocimetry, PIV) as well as computationally. For Reynolds numbers equal to or higher than 6,000 , the average velocities and velocity fluctuation levels scale well with the impeller tip speed, i.e. show Reynolds independent behavior. Surprising flow structures were measured - and confirmed through independent experimental repetitions - at Reynolds numbers around 1,300. Upon reducing the Reynolds number from values in the fully turbulent regime, the trailing vortex system behind the impeller blades weakens with the upper vortex weakening much stronger than the lower vortex. Simulations with a variety of methods (direct numerical simulations, transitional turbulence modeling) and software implementations (ANSYS-Fluent commercial software, lattice-Boltzmann in-house software) have only partial success in representing the experimentally observed laminar - turbulent transition.
\end{abstract}

Keywords: transitional flow; stereoscopic particle image velocimetry; computational fluid dynamics; stirred tank; Rushton turbine 


\section{Introduction}

Stirred tanks are basic pieces of equipment that are extensively used in industrial processes, among many more in fermentation and biopharmaceutical engineering. ${ }^{1}$ Whereas the majority of industrial mixing applications are in the turbulent flow regime, processes in the transitional regime (a regime in between laminar and fully turbulent) are gaining more and more attention, which is of fundamental importance for understanding the processes, the reactors, and related scale-up or scale-down.

Flow structures near the impeller are very complex: they are inherently three-dimensional and time dependent with periodic fluctuations due to impeller blade passages, as well as incoherent, non-periodic, turbulent fluctuations. Since impeller motion is the source of all flow in the mixing tank, it is worthwhile to investigate the flow near the impeller in detail. Many studies have concentrated on the fully turbulent flow regime ${ }^{2-5}$; much less is known about the transitional regime. Given the importance of up and down scaling processes and also given our interest in the way the laminar turbulent transition takes place, this paper focuses on the near-impeller flow in the transitional regime, with excursions to turbulent as well as laminar flow.

In mixing tanks, the Reynolds number is usually based on the impeller diameter $D$ as the length scale, $N D$ as the velocity scale ( $N$ is the angular velocity of the impeller in $\mathrm{rev} / \mathrm{s}$ ): $\operatorname{Re}=N D^{2} / v$ with $v$ the kinematic viscosity of the liquid (that we consider as being Newtonian and incompressible). Time-averaged velocity fields of stirred tank flow at high Reynolds numbers show Reynolds-independent behavior: average velocities and velocity fluctuation levels (quantified by root-mean-square values of the fluctuations) are to a good approximation independent of the Reynolds number when scaled with an appropriate macroscopic velocity scale (usually this is taken as the impeller tip speed $\mathrm{v}_{\text {tip }}=\pi N D$ ). ${ }^{3,6}$ One objective of our research is to investigate below what Reynolds number the average flow characteristics cease to be Reynolds independent.

The emphasis of this paper is on Particle Image Velocimetry (PIV) experiments on the flow driven by a Rushton turbine revolving in a cylindrical tank with baffles fitted at its perimeter. This is a de-facto standard stirred tank configuration that has been the subject of many experimental as well as computational research studies. ${ }^{6-9}$ Over the years, the experimental work has moved towards non-intrusive flow measurements that are able to probe local liquid velocities. In that respect, research on stirred tank flows has benefitted much from the availability of PIV instruments. ${ }^{10,11}$ Classical 2-D PIV captures the in-plane velocity components in an illuminated plane. By observing the illuminated plane with two cameras instead of one, the third, out-of-plane, velocity component can be recovered as well. This Stereoscopic PIV (SPIV) approach thus allows for estimating the turbulent kinetic energy (TKE) and the way it is distributed in a plane. Only few studies have used 
SPIV in turbulent mixing tanks: it has been employed to investigate the flow induced by a Rushton turbine ${ }^{6,12}$ and by a sawtooth impeller. ${ }^{13}$ It has - as of yet - not been used for transitional flow in mixing tanks.

Our experimental study has been complemented by numerical simulations in an attempt to enhance our understanding of the PIV results. In addition, we want to compare different numerical as well as modeling approaches. We have performed Direct Numerical Simulations (DNS) with commercial software (ANSYS-Fluent) as well as with in-house software based on the Lattice-Boltzmann Method (LBM). ${ }^{14,15}$ Given our focus on transitional flow we also tested turbulence modeling specifically designed for the transitional regime ${ }^{16}$ : the Transition Shear Stress Transport model as implemented in ANSYS-Fluent. Such turbulence modeling - if effective and accurate - is very useful to alleviate the high computational demands associated to the fine grids needed in a DNS. The availability of high-fidelity PIV data is a unique opportunity for assessing the results obtained with the various computational approaches.

The primary aim of this paper is to experimentally explore the limits of Reynolds-independent behavior of the average velocity fields, and to observe how the near-impeller flow changes its structure over the transition from high Reynolds numbers (turbulent flow) to low Reynolds numbers (laminar flow). A secondary aim is to assess the performance of computational approaches applied to this specific flow system.

This paper is organized in the following manner: in the next section we define our flow configuration: its geometry, liquids used, and operating conditions. Furthermore, the two PIV setups used will be described, along with the way PIV data have been analyzed. We then briefly discuss the computational approaches - and include extensive referencing to the literature - as well as how they have been used to simulate this specific flow. In the Results section, the emphasis is on the experimental results including an assessment of their accuracy and reproducibility. Subsequently numerical results are compared with experimental ones. The last section provides conclusions and suggestions for future work.

\section{Experimental setup}

The PIV experimental setup and a detailed view of the mixing tank and impeller are given in Figures 1 and 2 respectively. The inner diameter of the stirred tank was $T=0.285 \mathrm{~m}$. From this all other dimensions can be derived with the information in Figure 2 (except for the thickness of the baffles which was $8 \mathrm{~mm}$ ). As can be seen in Figure 1, there are four baffles, equally spaced along the inner perimeter of the tank. The measurement plane at $10^{\circ}$ in front of the baffle was chosen to have a field of view not obscured or distorted by the baffles (see Figure 1).

Tap water and three kinds of silicone oil were used as the working fluids, as listed in Table 1. The slight variations in the dynamic viscosity per liquid are due to variations in the temperature at which the experiments were conducted. A pentagon tank filled with the same fluid was placed around the cylindrical tank to minimize refraction (see Figure 1). 
Table1. Operational conditions of the PIV experiments.

\begin{tabular}{|c|c|c|c|c|c|c|}
\hline $\begin{array}{c}\text { PIV } \\
\text { system }\end{array}$ & Analysis method & Liquid & $\begin{array}{l}\text { Density } \\
/ \mathrm{kg} \cdot \mathrm{m}^{-3}\end{array}$ & $\begin{array}{l}\text { Viscosity } \\
\text { /mPa.s }\end{array}$ & $\begin{array}{c}\text { Rotational speed } \\
\text { /rpm }\end{array}$ & $\begin{array}{l}\text { Reynolds } \\
\text { number }\end{array}$ \\
\hline \multirow[t]{8}{*}{ SPIV } & \multirow{8}{*}{$\begin{array}{c}\text { correlation on fixed } \\
\text { interrogation windows } \\
\text { (32x32 pixels) with } 50 \% \\
\text { overlap }\end{array}$} & \multirow[t]{2}{*}{ Water } & \multirow[t]{2}{*}{1000} & \multirow[t]{2}{*}{1.00} & 70.0 & 10,000 \\
\hline & & & & & 40.0 & 6,000 \\
\hline & & \multirow[t]{2}{*}{ Silicone oil 1} & \multirow[t]{2}{*}{910} & 5.43 & 103 & 2,600 \\
\hline & & & & 5.42 & 52.0 & 1,310 \\
\hline & & \multirow[t]{3}{*}{ Silicone oil 2} & \multirow[t]{3}{*}{954} & 28.5 & 136 & 680 \\
\hline & & & & 29.7 & 90.6 & 440 \\
\hline & & & & 28.1 & 54.0 & 270 \\
\hline & & Silicone oil 3 & 966 & 161.5 & 111 & 100 \\
\hline \multirow[t]{6}{*}{ 2-D PIV } & \multirow{6}{*}{$\begin{array}{c}\text { correlation on adaptive } \\
\text { interrogation windows } \\
\text { (minimum size: } 32 \times 32 \text { pixels, } \\
\text { grid step size: } 16 \times 16 \text { pixels) }\end{array}$} & \multirow[t]{5}{*}{ Silicone oil 1} & \multirow[t]{5}{*}{910} & 5.36 & 88.0 & 2,250 \\
\hline & & & & 5.35 & 78.0 & 2,000 \\
\hline & & & & 5.34 & 68.5 & 1,750 \\
\hline & & & & 5.32 & 58.5 & 1,500 \\
\hline & & & & 5.30 & 38.7 & 1,000 \\
\hline & & Silicone oil 2 & 954 & 29.9 & 273 & 1,310 \\
\hline
\end{tabular}

Two CCD cameras (PowerView Plus, 4008×2672 pixels fitted with Micro Nikkor $60 \mathrm{~mm}$ lenses, Nikon, Japan) were placed perpendicularly to two faces of the pentagon tank so as to arrange the SPIV system. Camera B was placed such that it satisfied the Scheimpflug condition, which requires the measurement, image, and lens principal planes to intersect at a common point. ${ }^{6}$ The optical axis of the front lens of Camera B was at an angle of $50^{\circ}$ with the measurement plane; the optical axis of the lens of Camera A was at $90^{\circ}$. Therefore the offset half-angle between the two axes of both cameras was at $20^{\circ}$ in this work. This can effectively balance the non-uniform magnification with in and out-of-plane errors. ${ }^{17}$ In the 2-D PIV experiments reported in this paper, the layout as shown in Figure 1 was used with Camera B not operational. The SPIV experiments used a correlation method with fixed interrogation windows uniformly distributed over the field of view. The size of the interrogation windows were $32 \times 32$ pixels with $50 \%$ overlap, which led to a vector resolution of about $0.85 \mathrm{~mm}$. The 2-D PIV experiments used an adaptive interrogation windows method (which iteratively adjusts the size and shape of the individual interrogation areas in order to adapt to local seeding densities and flow gradients). ${ }^{18}$ The minimum size of the interrogation windows were $32 \times 32$ pixels, and the grid step sizes were $16 \times 16$ pixels, resulting in a vector resolution of about $0.45 \mathrm{~mm}$. To prevent the fluctuating velocities from being affected artificially, the raw instantaneous vectors were not subjected to any validation method; the erroneous vectors were directly deleted during the calculation of mean and fluctuating velocities. The ratio of effective samples to total samples near the impeller region were checked and greater than $96 \%$.

The illumination was provided by a dual pulsed laser system (DualPower, 325-15, 532nm, $325 \mathrm{~mJ}, 15 \mathrm{~Hz}$ ). The beam was converted by a spherical and a cylindrical lens to a laser sheet with a thickness of $1 \mathrm{~mm}$. Hollow glass microspheres (TSI, USA) with density of about $1500 \mathrm{~kg} / \mathrm{m}^{3}$ and with diameter of about 10 microns were used as seeding particles. Their 
concentration was adjusted to ensure that around 10-12 particle image pairs could be detected in one interrogation window. The time interval between two frames in an image pair is determined to ensure that the maximum in-plane and out-of-plane displacements of seeding particles are less than $1 / 4$ of the length of the interrogation window and $1 / 4$ of the thickness of the laser sheet.

The PIV system was triggered by an encoder (Kubler, Germany) that keeps track of the angular position of the impeller (angle $\theta$, see Figure 1 ) so that flow fields at specific angular positions relative to the impeller blades can be measured. Average velocity fields from $\theta=0^{\circ}$ to $50^{\circ}$ in steps of $10^{\circ}$ have been measured (note the $60^{\circ}$ periodicity given the 6 blades on the impeller). For each experimental condition, 400 pairs of images were captured for each of the 6 angels $\theta$ so as to obtain converged impeller angle-resolved average velocity data. Figure 3 shows the statistical convergence of the angle-resolved $\left(\operatorname{Re}=1,310, \theta=20^{\circ}\right)$ average velocities and their fluctuation levels at a position in the lower vortex structure $(2 z / W=0, r / R=1.2$, see Figure 7 later). If we compared average velocity data based on 400 image pairs with those based on 300 image pairs, deviations in average velocities and fluctuation levels were less than $2 \%$. For the average data based on 400 image pairs and those based on 200 images pairs, the deviations were less than $6 \%$.

With the different working fluids and with variations of the impeller speed we were able to cover a Reynolds number range between $10^{4}$ and $10^{2}$, see Table 1. A motor (ABB) was used to drive the impeller, and a frequency converter (Danfoss, Denmark) was used to control the rotational speeds within $\pm 0.5 \mathrm{rpm}$ accuracy. Moreover, the shaft and the Rushton turbine were carefully manufactured to prevent any apparent oscillation: the amplitude of oscillation was less than $0.1 \mathrm{~mm}$.

\section{Numerical simulations}

\section{Direct numerical simulation}

Given the modest Reynolds number values (see Table 1), in the first instance we have numerically solved the incompressible Navier-Stokes equations and the continuity equation without any form of turbulence modeling. Grid requirements for such "direct" simulations have been estimated by relating the Kolmogorov length scale $\eta$ to a macroscopic length scale (for which we take the impeller diameter $D$ ) through the Reynolds number: $\eta=D \cdot R e^{-3 / 4}$. For the highest Reynolds number of $10^{4}$, and with an impeller diameter of $95 \mathrm{~mm}, \eta=95 \mu \mathrm{m}$. A typical criterion for performing DNS is $\Delta \leq \pi \eta$ with $\Delta$ the grid spacing. ${ }^{19,20}$

In our "direct" simulations the typical grid spacing was $800 \mu \mathrm{m}$. It means that our grid was too coarse for DNS for the highest Reynolds numbers. However, for $\mathrm{Re} \leq 2,600$ the grid was sufficiently fine for DNS. It should be noted that the 
above are only rough estimates of resolution requirements. Turbulence (or transitional flow) in the tank is very inhomogeneous so that the sizes of the finest dynamic lengths scales will vary significantly over the tank volume.

\section{Transitional turbulence modeling}

To alleviate grid resolution requirements, turbulence modeling is an alternative for direct simulations. Most turbulence modeling has been based on notions that hold for fully developed turbulent flows only. Given our interest in transitional flows, one of the aims of this paper is to assess the performance of a turbulence model that has been proposed specifically for transitional flow: the Transition Shear-Stress Transport-model. ${ }^{16}$ The Shear-Stress Transport (SST) model is a well-established turbulence model. ${ }^{21}$ It is based on a $k-\omega$ formulation, i.e. it solves transport equations for the turbulent kinetic energy, and the specific dissipation. It switches - dependent on wall proximity - between the $k-\omega$ model of Wilcox ${ }^{22}$ and the (standard) $k-\varepsilon$ model via a blending function. In addition, it features a novel formulation as compared to the $k-\varepsilon$ model as well as the $k-\omega$ model - of the eddy viscosity based on shear stress transport. ${ }^{21}$

In the Transition SST model, additional transport equations for intermittency and for the transition momentum thickness Reynolds number are solved. ${ }^{16}$ These new variables interact with the SST model via the destruction and production terms in the transport equation for TKE, as well as with the blending function.

Full details of the Transition SST model can be found in Menter. ${ }^{16}$ In a subsequent paper, Langtry et al. ${ }^{23}$ successfully validated the Transition SST model with a number of turbomachinery test cases, including transient, three-dimensional flow. In our research we have used the same model coefficients as the ones specified in Menter. ${ }^{16}$

\section{Computational aspects}

The geometrical configuration used for the CFD simulation in this work was the same as that used for the PIV experiments.

The DNS's have been performed with two different computer codes. In the first place, we have a code based on the lattice-Boltzmann method (LB-DNS). ${ }^{14,15}$ This code, which has been used extensively for laminar and turbulent flows in agitated tanks, ${ }^{24-27}$ uses a uniform, cubic grid with $360^{3}$ cells. The spatial resolution is such that the diameter of the mixing tank spans 360 grid spacings $\Delta: T=360 \Delta$. As for the temporal resolution, it takes 3600 time steps for one impeller revolution. The code uses an immersed boundary method to represent the (moving) no-slip boundary conditions at the surface of the impeller, as well as at the (static) baffled tank wall. To collect impeller angle-resolved average flow data under quasi steady conditions, first the simulations ran over 40 impeller revolutions starting from a zero-velocity liquid to develop the flow. Then 30 subsequent impeller revolutions were used to collect flow data to determine average velocities 
and average (root-mean-square) velocity fluctuation levels.

In the second place, DNS's were performed with the commercial code ANSYS-Fluent 14.5. These DNS's are termed FV-DNS (since they are based on the finite-volume method). These simulations use a non-uniform, hexahedral grid with about 4.7 million cells. In the impeller region a fine grid was utilized with almost the same resolution as the cubic LB grid (where the LB grid had 24 grid spacings over the blade height, the FV grid had 22 spacings). To represent the revolving impeller, the FV-DNS simulations have used the multiple-reference frame approach ${ }^{28}$ at $R e=270$, and the sliding mesh method $^{29}$ at $\operatorname{Re}=1,310$ and $\operatorname{Re}=2,600$. The temporal resolution of the simulations with sliding mesh method is such that one impeller revolution takes 720 time steps. The second order upwind scheme was used for the spatial discretization of the momentum equations, and the second order implicit scheme for time advancement. The FV-DNS with sliding mesh approach could reach quasi-steady state within 20 impeller revolutions because it started from a converged steady state flow field calculated by the Transition SST model. Then average flow fields were obtained by collecting data and averaging the data over subsequent 20 impeller revolutions, which shows good statistical convergence.

The same grid as used for the FV-DNS was also used for the simulations with the Transition SST turbulence model. These simulations used the multiple-reference frame approach for representing the revolving impeller and second order upwind scheme for the spatial discretization of all the equations. At $R e=1,310$ Transition SST simulations with the multiple-reference method and the sliding mesh method were compared and showed very close agreement. The Transition SST model is a standard feature in ANSYS-Fluent 14.5.

ANSYS-Fluent solves the transport equations in an iterative manner. It was ensured that every normalized residual fell below the specified convergence tolerance of $1 \times 10^{-4}$, for a well-converged simulation. The velocities at three different positions near the impeller region, as well as the torque acting on the impeller, were monitored to judge the convergence of the simulations.

\section{Results and discussion}

In the following discussion of PIV and numerical results, the radial, axial, tangential mean velocities and TKE are represented by $U_{r}, U_{z}, U_{t}$, and $k$, respectively. The fluctuating velocity components (in terms of root-mean-square values) are represented by $u_{r}^{\prime}, u_{z}^{\prime}$, and $u_{t}^{\prime}$, respectively. All velocities have been normalized with the impeller tip velocity $V_{\text {tip, }}$ and $k$ has been normalized with $V_{\text {tip }}{ }^{2}$. The origin of the coordinate system is located at the center of the turbine disk (see Figure 2). The axial coordinate is normalized with the half width of the blade $W / 2$ and denoted by $2 z / W$; the radial coordinate is normalized with the radius of the turbine $R=D / 2$ and denoted by $r / R$. 


\section{Comparison between SPIV and 2-D PIV results under turbulent conditions}

As a result of the above-mentioned arrangement of the two cameras in our SPIV experiment, the two kinds of PIV systems can be operated simultaneously, and their results can be compared directly. As presented in Figure 4 (top row), the velocity fields obtained from the stereo- and the 2-D PIV data in this work show good qualitative agreement. Also the structure of the TKE distribution is very similar for the two PIV setups (see bottom row of Figure 4) with high turbulence levels associated to the vortex cores. Such effects have been earlier identified by several researchers, e.g., Refs. 5 and 30. Care should be taken when comparing TKE levels between 2-D PIV and SPIV since in the 2-D experiments the out-of-plane (i.e. tangential) velocity component is not available; in the SPIV it is. It also has to be emphasized that these are phase-resolved turbulent kinetic energy levels based on instantaneous velocity measurements all taken at the same impeller angle (of $\theta=40^{\circ}$ ) relative to the measurement plane. This kinetic energy therefore does not contain a contribution from periodic (blade-passage) fluctuations, it only contains turbulent fluctuations.

For a quantitative comparison we show part of the data of Figure 4 in Figure 5 as radial profiles of radial and axial average velocity at two different vertical locations $(2 z / W=0$ is the center of the impeller, $2 z / W=1$ is the top of the impeller), and of radial and axial fluctuating velocity components at $2 z / W=1$. The results with the two PIV approaches show close agreement. The vector resolutions obtained by the two PIV system are different. To present a similar vector density in Figure 4 and data point density in Figure 5, we have skipped one of every two successive 2-D PIV data points in each direction.

In Figure 6 we compare current results at $\operatorname{Re}=10,000$ and 6,000 for the average tangential velocity with those obtained previously under fully turbulent conditions at $\operatorname{Re}=40,000^{6}$ (where it should be noted that the results from Ref. 6 were obtained for an impeller with blade thickness of $t / D=0.01$ where in the current study it is $t / D=0.02$ ). We observe an overall agreement which indicates Reynolds independence at least down to $\operatorname{Re}=6,000$. The minor differences close to the impeller

(for $1.0<r / R<1.2$ ) between $\operatorname{Re}=40,000$ on one side, and $\operatorname{Re}=10,000$ and 6,000 on the other we attribute to the slight geometrical differences.

In summary, the in-plane flow characteristics obtained by the two PIV systems (2-D and stereoscopic) show close agreement. The average out-of-plane (tangential velocity) shows reasonable agreement to the previous study ${ }^{6}$, and also indicates that Reynolds number independence of the scaled, average velocities is sustained down to at least $\operatorname{Re}=6,000$.

\section{Transitional flow results}

Figure 7 summarizes some of the key findings of this paper. Here we show - in terms of average velocity vectors - the 
evolution of the trailing vortex structure that develops in the wake of an impeller blade when the Reynolds number is gradually reduced. In the top row of the figure one does not observe significant changes when Re is brought down from 10,000 to 6,000 . This is consistent with the results in Figure 6 for the tangential velocity. At $R e=2,600$, and more pronounced at $\mathrm{Re}=2,000$ we see a severe weakening of the upper vortex while the lower vortex gets significantly stronger, specifically at $\operatorname{Re}=2,000$. At the next Reynolds number investigated, $R e=1,310$, the upper vortex has virtually disappeared. Further lowering the Reynolds number results in a more and more weaker lower vortex until (at $\operatorname{Re}=100)$ there is hardly a trailing vortex system and the liquid is simply pushed in radial direction by the impeller blade in an almost top-bottom symmetric manner. To the best of our knowledge, this transition scenario between $\operatorname{Re}=6,000$ and $\operatorname{Re}=100$ has not been observed before. Our observations are consistent with the seminal work by van't Riet and Smith ${ }^{31}$, who measured a strongly weakened vortex structure at $\operatorname{Re}=300$ and Reynolds independence for $\operatorname{Re}>=15,000$.

In order to check for possible artifacts, the experiment as reported in Figure 7 at $\mathrm{Re}=1,310$ was repeated with a different silicon oil: Silicon oil 2 (see Table 1) with a dynamic viscosity $\mu=29.9 \mathrm{mPa} \cdot \mathrm{s}$ (instead of Silicon oil 1 with $\mu=5.42 \mathrm{mPa} \cdot \mathrm{s}$ as was used to generate Figure 7 for $\mathrm{Re}=1,310)$. At the same Reynolds number of 1,310 the vortex structure is very similar for the two liquids, see Figure 8 . Figure 9 shows profiles extracted from the data of Figure 8 to also show the levels of agreement for different oils at the same Reynolds number in a quantitative sense. In addition, Figure 9 demonstrates good agreement of velocity fluctuation levels as measured in the two liquids.

After having shown qualitative observations of an interesting transition from turbulent to laminar flow associated to the trailing vortex system we now show sets of radial velocity profiles at the mid-height of the impeller. In the top row of Figure 10 we see that the scaled velocities at $\operatorname{Re}=10,000$ and 6,000 are very similar, and are deviating from those at $\operatorname{Re}=2,600$. These deviations get very pronounced when Re is further reduced as can be observed in the bottom row of Figure 10. There one sees that upon reducing the Reynolds number, the axial velocities get reduced to almost zero and that radial velocities show less pronounced radial profiles. Where there is a clear trend of radial and axial velocity profiles with the Reynolds number, this is less so the case for the average tangential velocity, see Figure 11. The highest tangential velocity occurs at the intermediate Reynolds number of 680 ; the radial decay of the tangential velocity is slowest for $\operatorname{Re}=270$ and fastest for $\operatorname{Re}=1,310$.

Results for velocity fluctuations have been summarized in Figure 12. These are radial profiles of phase-resolved turbulent kinetic energy (which only contains random contributions, no periodic contributions). All results in Figure 12 have been obtained with the SPIV setup so that $k$ contains contributions from all three velocity components. The most significant overall drop in TKE occurs near $\mathrm{Re}=1,310$ which (see Figure 7 ) is also where the most significant change in the trailing vortex structure occurs. Only at $\mathrm{Re}=270$ the fluctuation levels become virtually zero over the entire radial range 
investigated.

The difference in strength of the upper and lower vortex at Re=1,310 is evident from Figure 13 that, next to velocity vectors, shows the vorticity associated with the average flow; more specifically its component normal to the plane of view: $\omega=\frac{\partial U_{z}}{\partial r}-\frac{\partial U_{r}}{\partial z}$. It can be seen that vorticity is being generated in the near wake of the blade, near the upper and lower blade edge (Figure 13 at $10^{\circ}$ ). The peak-vorticity of the lower vortex is a factor of two higher than that of the upper vortex. The vorticity gradually decays at larger angles behind the blade. In the vicinity of the vortices, relatively high levels of turbulent kinetic energy have been recorded (Figure 14). TKE in the lower vortex core decays slower with increase of impeller angle than vorticity: at $\theta=50^{\circ}$, high TKE levels are still observed whereas vorticity has already decayed to $50 \%$ of its peak levels.

\section{Prediction of transitional flow characteristics in the stirred tank}

We now will be investigating if computational approaches are able to reproduce the key features of the turbulent / laminar transition as were observed by means of the PIV experiments. In this respect we are focusing on three Reynolds numbers: (1) $\operatorname{Re}=2,600$ is the Reynolds number at and below which Reynolds independence ceases to exist (see Figures 7 and 10); (2) $R e=1,310$ which has the strongest asymmetric vortex structure (see Figure 7); (3) $R e=270$ where velocity fluctuations are almost absent (see Figure 12).

The PIV results show that at $\mathrm{Re}=2,600$ we are not in the fully turbulent, Reynolds-independent regime. For that reason, we tested the Transition SST model and FV-DNS for this case, see Figure 15. There is very good agreement between experiments and both simulations in terms of the (impeller angle-resolved) average velocity field. Specifically the FV-DNS is well able to predict the strength of average vorticity, and the way the trailing vortices move away from the impeller blade and dissipate: the location (at $r / R \approx 1.6$ ) and strength of the vortices associated to the previous blade passage agree well with the PIV experiments. Predictions of TKE are less good. The Transition SST model shows a significant overprediction of TKE; the FV-DNS an underprediction. The overprediction of TKE by a RANS-based turbulence model is remarkable. In fully developed turbulent stirred tank flow, RANS-based models tend to underpredict TKE, e.g., Ref. 8. The lower levels of TKE in the FV-DNS might have to do with spatial resolution not being sufficiently high. We leave this issue for future studies.

The case $\mathrm{Re}=1,310$ has been numerically investigated with all the approaches available to us: Transient SST, FV-DNS, and LB-DNS. None of these simulation methods is able to accurately represent the strongly asymmetric vortex structure as measured with PIV, see Figure 16. The two DNS methods do show an asymmetric vortex system with a more pronounced 
lower vortex, and a weaker upper vortex, however by far not to the extent as measured by PIV. The LB-DNS result shows a lower vortex that is somewhat stretched in the radial direction. Where the lower vortex in the PIV is circular, the one in the LB-DNS simulation is ellipsoidal. The two DNS's shows the remnants of the lower vortex shed off the preceding impeller blade at $r / R \approx 1.6$. This remnant can also be seen in the PIV result at approximately the same location.

The Transition SST prediction does not show an asymmetric vortex system. The result at $\operatorname{Re}=1,310$ is similar to the Transition SST result for $\mathrm{Re}=2,600$ as shown in Figure 15. The Transition SST model seems unable to capture the Reynolds number effects as observed in the experiments. It has to be noted, however, that given the weak velocity fluctuation levels as observed in Figure 12, the flow at $\mathrm{Re}=1,310$ should likely be characterized as laminar, not transitional, so that application of the Transition SST model is not that appropriate for the $\mathrm{Re}=1,310$ case.

The poor performance of the simulations at $\mathrm{Re}=1,310$ in terms of average velocity imply that also velocity fluctuation levels are not accurately predicted. An important source of turbulence generation are gradients in the average flow. Since we cannot get the latter right, turbulence levels are not well predicted; compare the simulated distributions of TKE with those measured (the right panels of Figure 16).

It is shown in Figure 17 that the ANSYS-Fluent code is well able to deal with the laminar flow at Re=270. The numerical predictions of the radial profiles of average radial and axial velocity are in good agreement with the PIV results.

As an additional assessment of the simulations, the power number with FV-DNS and Transition SST model are compared with the experimental data from Chapple et al. ${ }^{32}$, see Figure 18 . The power number $N_{\mathrm{p}}$ was calculated as $N_{\mathrm{p}}=2 \pi \frac{M}{\rho N^{2} D^{5}}$ with $M$ the torque acting on the Rushton turbine. The simulated results for the power number show a fairly good agreement with the experimental data, also in situations ( $R e=1,310$ and 2,600 ) where the flow field predictions show significant deviations from PIV experiments.

\section{Conclusions}

PIV experiments were conducted to study how the flow near the impeller blades in a mixing tank goes through a laminar-turbulent transition. The impeller used was a Rushton turbine - a de-facto standard in mixing research - which has a characteristic trailing vortex structure associated to it. It was observed that this structure strongly depends on the Reynolds number. For $\operatorname{Re} \geq 2,600$ a structure as extensively observed in the literature was measured: an upper and a lower vortex form in the wake of each impeller blade and are swept radially outward and dissipated in the tank volume. This structure is responsible for much of the turbulence generated in the tank and therefore important for the mixing performance of this agitator. For Reynolds numbers below 2,600, a peculiar transition occurred: the strength of the lower 
vortex increases whereas that of the upper vortex decreases. This effect reaches its pinnacle at $\operatorname{Re}=1,310$ where the upper vortex is virtually absent and the lower vortex is very strong (the dimensionless vorticity in its core is almost two times larger than at $\operatorname{Re}=2,600)$. Below $\operatorname{Re}=1,310$ the lower vortex gradually weakens and when $\operatorname{Re}=100$ is reached the flow generated by the impeller blades is a simple flow with the stream coming off the impeller in radial direction.

In addition to the vortex structure being strongly dependent on the Reynolds number, we observed other Reynolds number effects: Down to $\mathrm{Re}=6,000$ the velocity field shows to a good approximation Reynolds independent behavior, i.e. scaled - with the impeller tip speed - average velocities and velocity fluctuation levels are independent of the Reynolds number. This Reynolds independency has ceased to exist at Re=2,600. Velocity fluctuations - other than periodic fluctuations caused by impeller blade passage - virtually disappear if $\operatorname{Re} \leq 270$.

Overall, our study thus identifies a turbulent-to-transitional flow transition between $\operatorname{Re}=6,000$ and 2,600 characterized by loss of Reynolds independency, and a transitional-to-laminar transition at $\mathrm{Re} \approx 270$ characterized by disappearance of random velocity fluctuations. The transitional region shows a drastic change in the structure of the trailing vortex system.

We had limited success in representing this peculiar transition with numerical flow simulations. Because of this, and also since this effect has - as far as we are aware - not been reported in the literature, we have thoroughly checked our experimental system to see if there was an artifact causing this. Geometrical imperfections were kept to a minimum. As an example, eccentricities of the shaft and impeller were less than $0.1 \mathrm{~mm}$ (with an impeller diameter of $95 \mathrm{~mm}$ ). We also confirmed that the effect was purely a Reynolds number effect: liquids with different viscosity (by a factor of 5) but agitated at the same Reynolds number produced the same dimensionless velocity fields. Finally, the results were largely independent of the particular PIV analysis technique.

Direct numerical simulation (DNS) approaches were successful in predicting the near-impeller average flow at Re=2,600 and $\operatorname{Re}=270$. At $\operatorname{Re}=2,600$ velocity fluctuation levels were, however, underpredicted by the DNS. A turbulence model specially designed for transitional flow (Transition SST model) was less successful at predicting the average flow as well as fluctuation levels at $\mathrm{Re}=2,600$.

Numerical simulations were not well able to reproduce the case of $R=1,310$. DNS methods did predict an asymmetric vortex system, however not to the extent as experimentally observed. Reasons for these discrepancies are not known and need further investigation. We do remark that different direct simulation approaches gave somewhat different results so that numerical / grid effects are the likely cause of the deviations with experiments.

\section{Acknowledgement}

The authors gratefully acknowledge the financial support from the National Natural Science Foundation of China 
(No.21376016).

\section{Literature Cited}

1. Chu L, Robinson DK. Industrial choices for protein production by large-scale cell culture. Curr Opin Biotechnol. 2001;12:180-187.

2. Derksen JJ, Doelman MS, Van den Akker HEA. Three-dimensional LDA measurements in the impeller region of a turbulently stirred tank. Exp Fluids. 1999;27:522-532.

3. Li Z, Bao Y, Gao Z. PIV experiments and large eddy simulations of single-loop flow fields in Rushton turbine stirred tanks. Chem Eng Sci. 2011;66:1219-1231.

4. Li Z, Hu M, Bao Y, Gao Z. Particle image velocimetry experiments and large eddy simulations of merging flow characteristics in dual Rushton turbine stirred tanks. Ind Eng Chem Res. 2012;51:2438-2450.

5. Khan FR, Rielly CD, Brown DAR. Angle-resolved stereo-PIV measurements close to a down-pumping pitched-blade turbine. Chem Eng Sci. 2006;61:2799-2806.

6. Li Z, Song G, Bao Y, Gao Z. Stereo-PIV experiments and large eddy simulations of flow fields in stirred tanks with Rushton and curved-blade turbines. AIChE J. 2013;59:3986-4003.

7. Escudie R, Line A. Experimental analysis of hydrodynamics in a radially agitated tank. AIChE J. 2003;49:585-603.

8. Hartmann H, Derksen JJ, Montavon C, Pearson J, Hamill IS, Van den Akker HEA. Assessment of large eddy and RANS stirred tank simulations by means of LDA. Chem Eng Sci. 2004;59:2419-2432.

9. Gimbun J, Rielly CD, Nagy ZK, Derksen JJ. Detached eddy simulation on the turbulent flow in a stirred tank. AIChE J. $2012 ; 58: 3224-3241$.

10. Escudié R, Bouyer D, Liné A. Characterization of trailing vortices generated by a Rushton turbine. AIChE J. 2004;50:75-86.

11. Sharp K V., Adrian RJ. PIV study of small-scale flow structure around a Rushton turbine. AIChE J. 2001;47:766-778.

12. Yoon HS, Hill DF, Balachandar S, Adrian RJ, Ha MY. Reynolds number scaling of flow in a Rushton turbine stirred tank. Part I - Mean flow, circular jet and tip vortex scaling. Chem Eng Sci. 2005;60:3169-3183.

13. Unadkat H, Rielly CD, Nagy ZK. PIV study of the flow field generated by a sawtooth impeller. Chem Eng Sci. $2011 ; 66: 5374-5387$

14. Somers JA. Direct simulation of fluid flow with cellular automata and the lattice-Boltzmann equation. Appl Sci Res. $1993 ; 51: 127-133$.

15. Chen S, Doolen GD. Lattice Boltzmann method for fluid flows. Annu Rev Fluid Mech. 1998;30:329-364. 
16. Menter FR, Langtry RB, Likki SR, Suzen YB, Huang PG, Völker S. A correlation-based transition model using local variables - Part I: Model formulation. J Turbomach. 2006;128:413-422.

17. Lawson NJ, Wu J. Three-dimensional particle image velocimetry: Experimental error analysis of a digital angular stereoscopic system. Meas Sci Technol. 1997;8:1455-1464.

18. Theunissen R, Scarano F, Riethmuller ML. An adaptive sampling and windowing interrogation method in PIV. Meas Sci Technol. 2007;18:275-287.

19. Moin P, Mahesh K. Direct numerical simulation: a tool in turbulence research. Annu Rev Fluid Mech. 1998;30:539-578.

20. Eswaran V, Pope SB. An examination of forcing in direct numerical simulations of turbulence. Comput Fluids. $1988 ; 16: 257-278$.

21. Menter FR. Two-equation eddy-viscosity turbulence models for engineering applications. AIAA J. $1994 ; 32: 1598-1605$.

22. Wilcox DC. Reassessment of the scale-determining equation for advanced turbulence models. AIAA J. $1988 ; 26: 1299-1310$.

23. Langtry RB, Menter FR, Likki SR, Suzen YB, Huang PG, Völker S. A correlation-based transition model using local variables-Part II: Test cases and industrial applications. J Turbomach. 2006;128:423-434.

24. Derksen JJ. Highly resolved simulations of solids suspension in a small mixing tank. AIChE J. 2012;58:3266-3278.

25. Mo J, Gao Z, Bao Y, Li Z, Derksen JJ. Suspending a solid sphere in laminar inertial liquid flow-experiments and simulations. AIChE J. 2015;61:1455-1469.

26. Derksen JJ, Van den Akker HEA. Large eddy simulations on the flow driven by a Rushton turbine. AIChE J. $1999 ; 45: 209-221$.

27. Gillissen JJJ, Van den Akker HEA. Direct numerical simulation of the turbulent flow in a baffled tank driven by a Rushton turbine. AIChE J. 2012;58:3878-3890.

28. Luo JY, Issa RI, Gosman AD. Prediction of impeller-induced flows in mixing vessels using multiple frames of reference. IChemE Symp Ser. 1994;136:549-556.

29. Bakker A, Laroche RD, Wang MH, Calabrese RV. Sliding mesh simulation of laminar flow in stirred reactors. Chem Eng Res Des. 1997;75:42-44.

30. Shekhar C, Nishino K, Yamane Y, Huang J. Stereo-PIV measurement of turbulence characteristics in a flow mixer. J Vis. 2012;15:293-308.

31. van't Riet K, Smith JM. The trailing vortex system produced by Rushton turbine agitators. Chem Eng Sci. 
1975;30:1093-1105.

32. Chapple D, Kresta SM, Wall A, Afacan A. The effect of Impeller and tank geometry on power number for a pitched blade turbine. Chem Eng Res Des. 2002;80:364-372. 
Figure 1

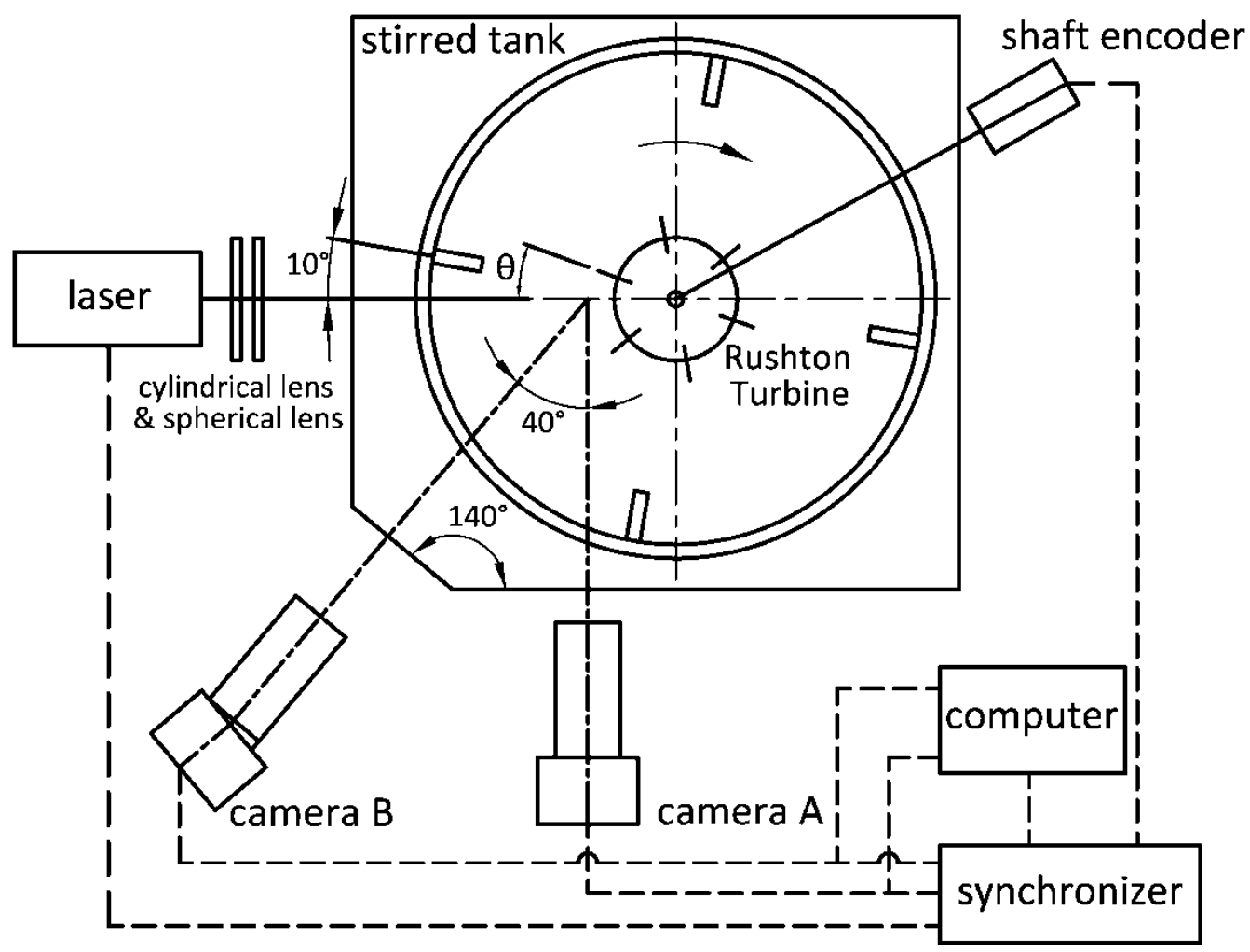

Figure 1.Experimental setup for the stereoscopic PIV (SPIV) system: two cameras view a plane in the flow illuminated by a laser sheet. In the 2-D PIV experiments only camera A is operational. By monitoring the impeller's angular location with the shaft encoder, we are able to measure angle-resolved velocity fields (the measured flow field is under an angle $\theta$ with respect to the impeller blade). The angle between baffle and measured flow field has been fixed to $10^{\circ}$. 
Figure 2
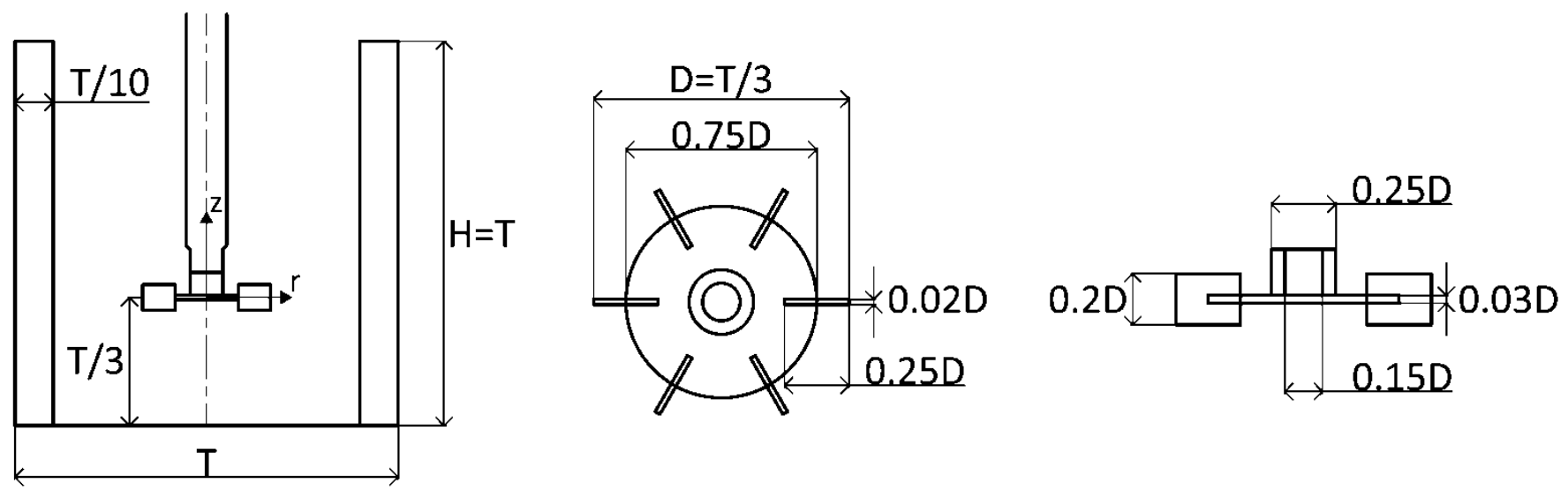

Figure 2. Flow geometry and $(r, z)$ coordinate system with its origin at the center of the impeller (left) and top (middle) and side view (right) of the impeller. The tank is filled with liquid up to a level $H=T$; the liquid has a free surface at the top. $T=285 \mathrm{~mm}$. 
Figure 3
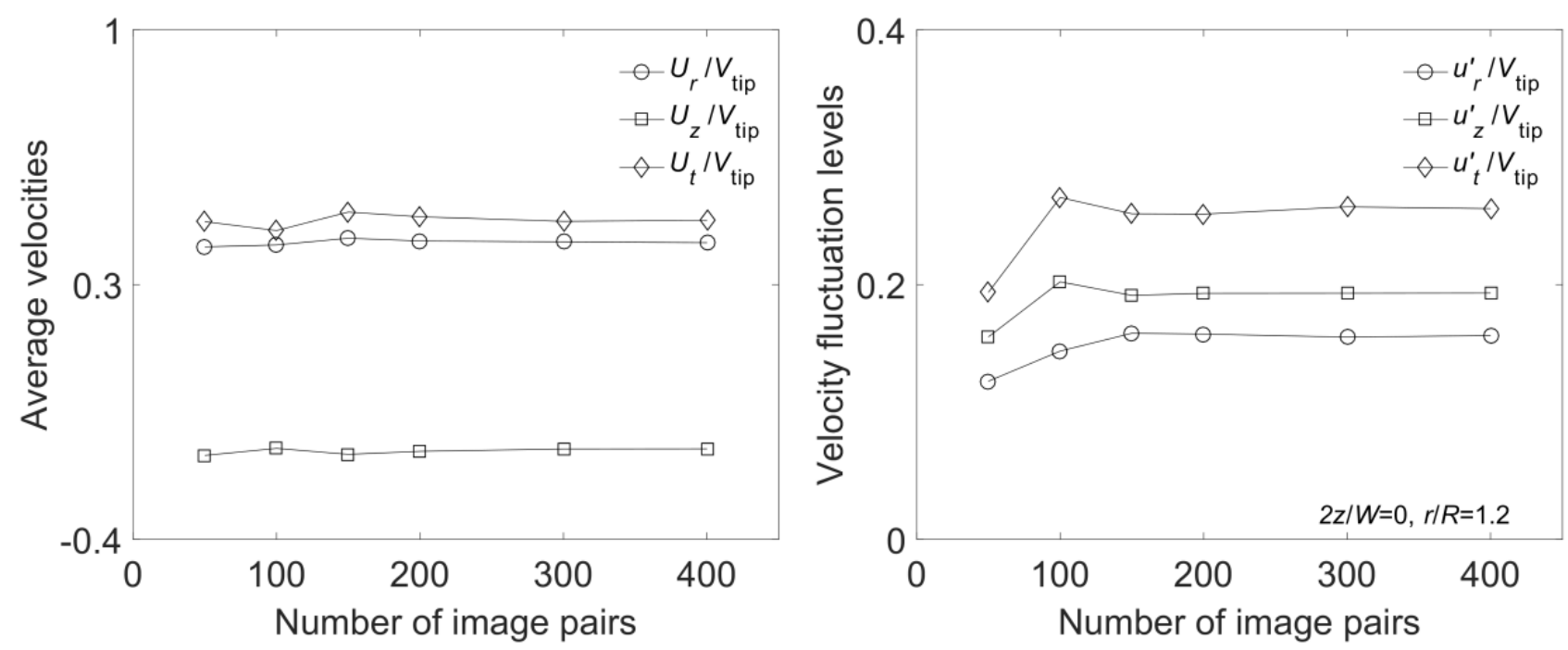

Figure 3. Average velocities (left) and fluctuation levels (right) as a function of the number of image pairs involved in the averaging process. SPIV at $2 z / W=0, r / R=1.2, R e=1,310$, and $\theta=20^{\circ}$. 

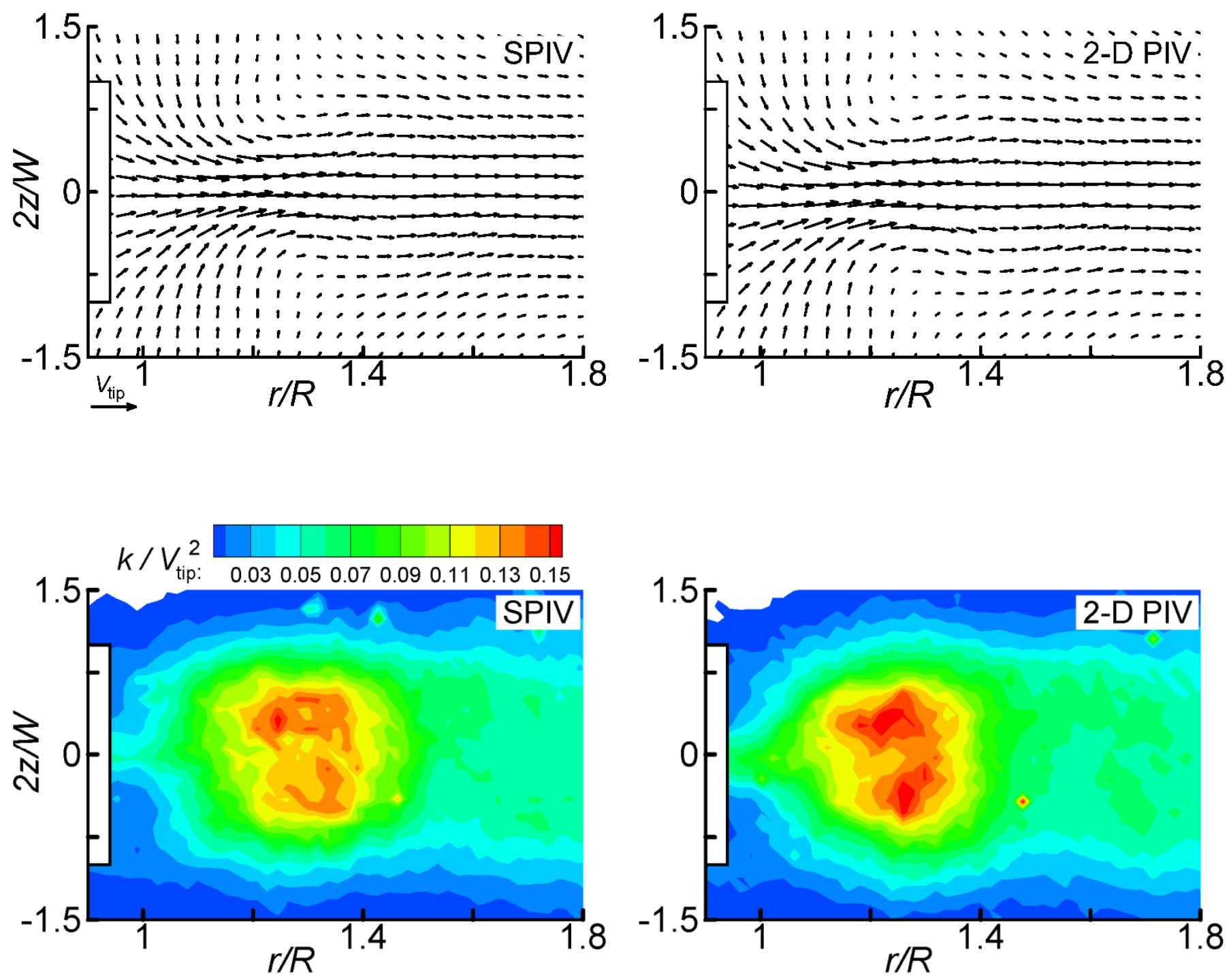

Figure 4. Phase-resolved average velocity vector field (top) and TKE distribution (bottom) at $\operatorname{Re}=6,000$ and $\theta=40^{\circ}$

obtained by SPIV and 2-D PIV systems simultaneously. In the 2-D PIV result, TKE has been determined from fluctuating radial and axial velocity components: $k=\frac{3}{4}\left(\overline{u_{r}^{\prime 2}}+\overline{u_{z}^{\prime 2}}\right)$; in SPIV from all three components:

$$
k=\frac{1}{2}\left(\overline{u_{r}^{\prime 2}}+\overline{u_{z}^{\prime 2}}+\overline{u_{t}^{\prime 2}}\right)
$$


Figure 5
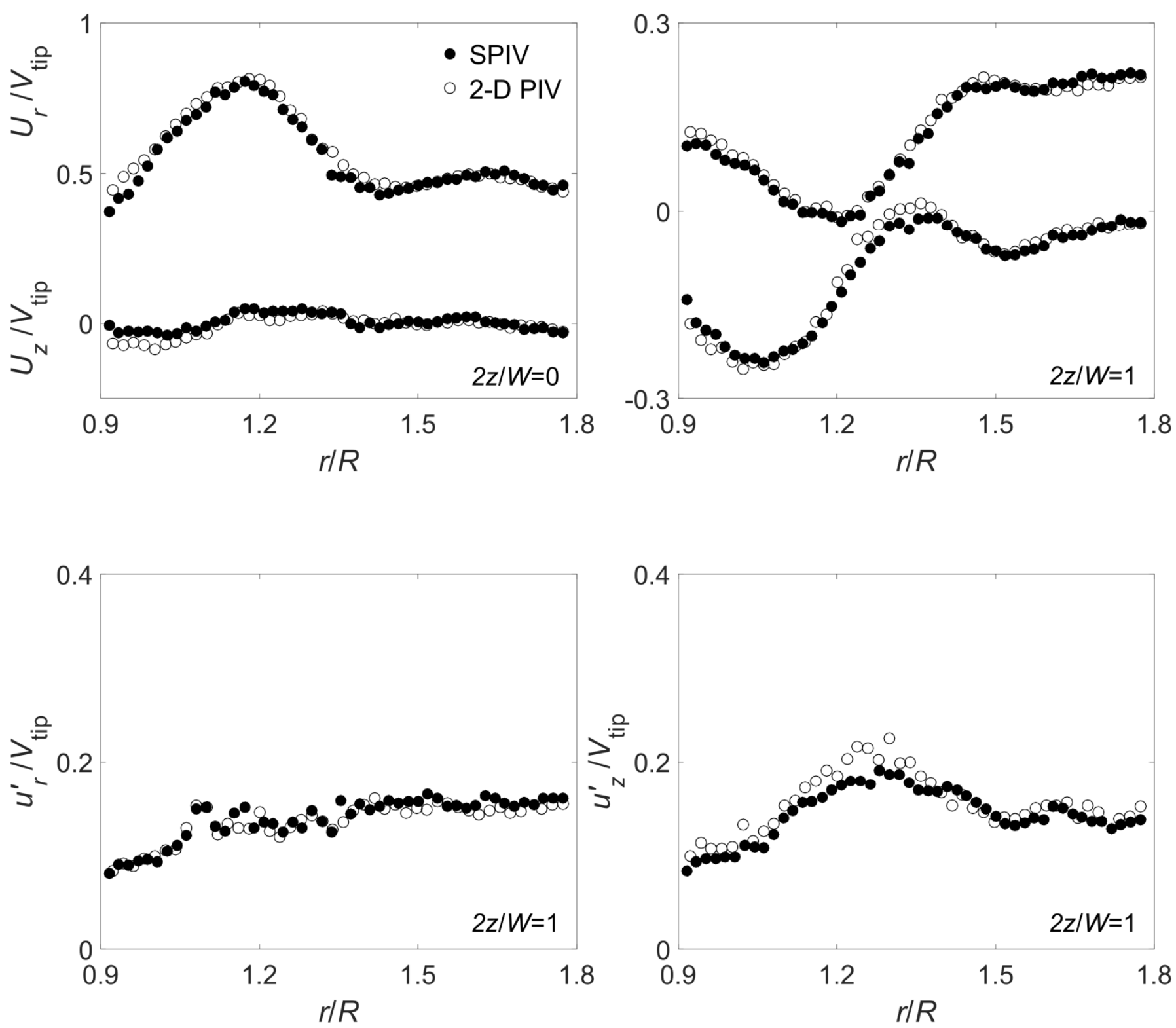

Figure 5. Comparison between SPIV and 2-D PIV results at $\operatorname{Re}=6,000, \theta=40^{\circ}$. Top row: axial $\left(U_{z}\right)$ and radial $\left(U_{r}\right)$ average velocities on two horizontal lines, $2 z / W=0$ and $2 z / W=1$; bottom row: axial $\left(u_{z}^{\prime}\right)$ and radial $\left(u_{r}^{\prime}\right)$ fluctuating velocity components at $2 z / W=1$. 
Figure 6

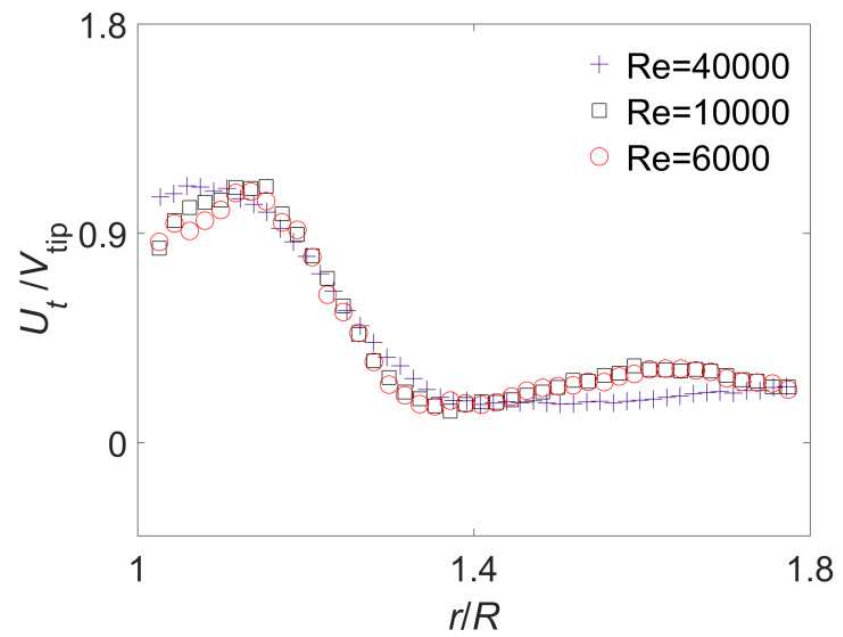

Figure 6. Radial profiles of the average tangential velocity at $2 z / W=0$ and $\theta=20^{\circ}$ measured with SPIV; comparison between different Reynolds numbers. The $\mathrm{Re}=40,000$ results were taken from Ref. 7 and were obtained in a slightly different mixing tank. 
Figure 7
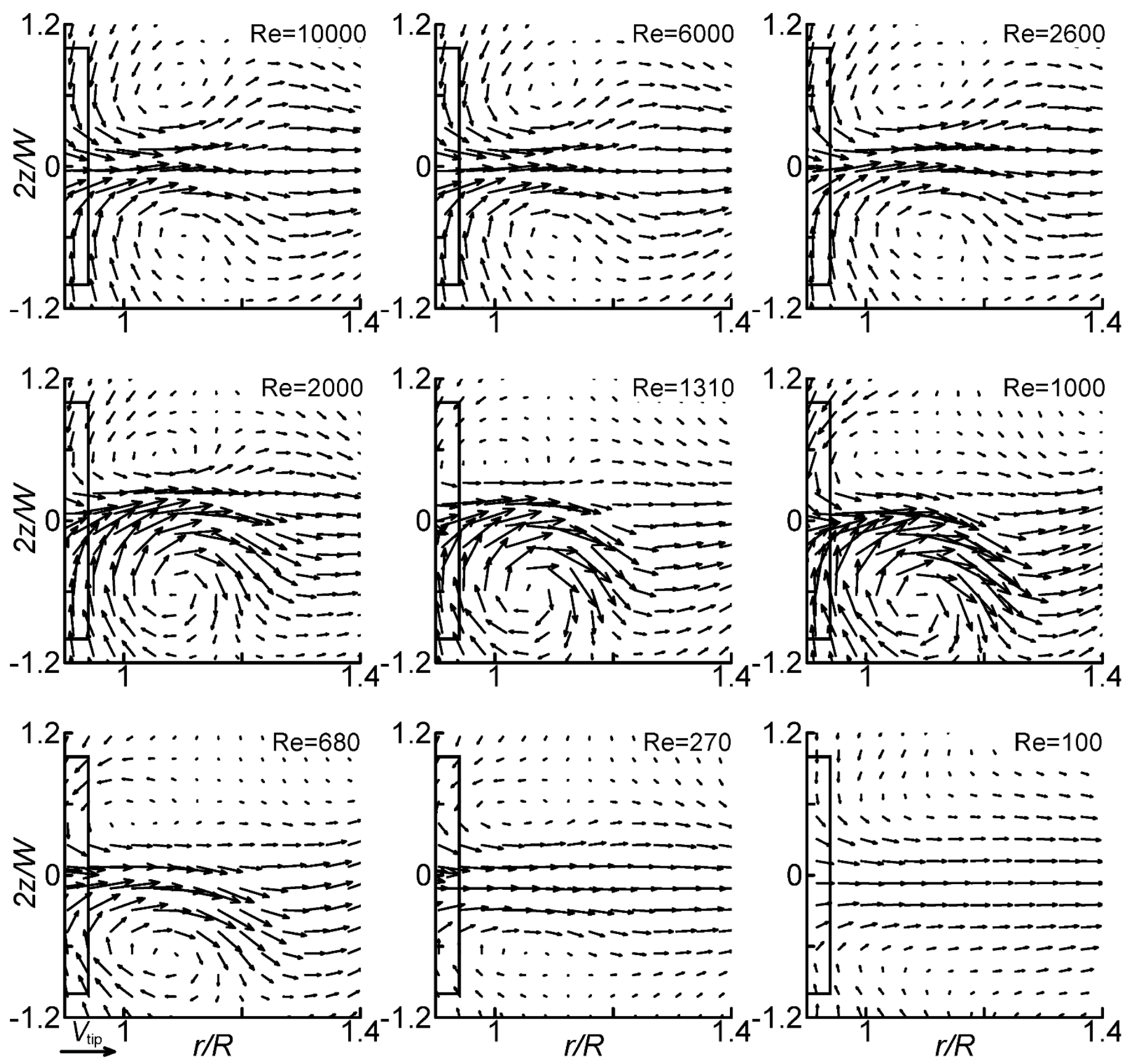

Figure 7. Phase-resolved average velocity fields at $\theta=20^{\circ}$ with Reynolds numbers ranging from 100 to 10,000 . PIV results. 

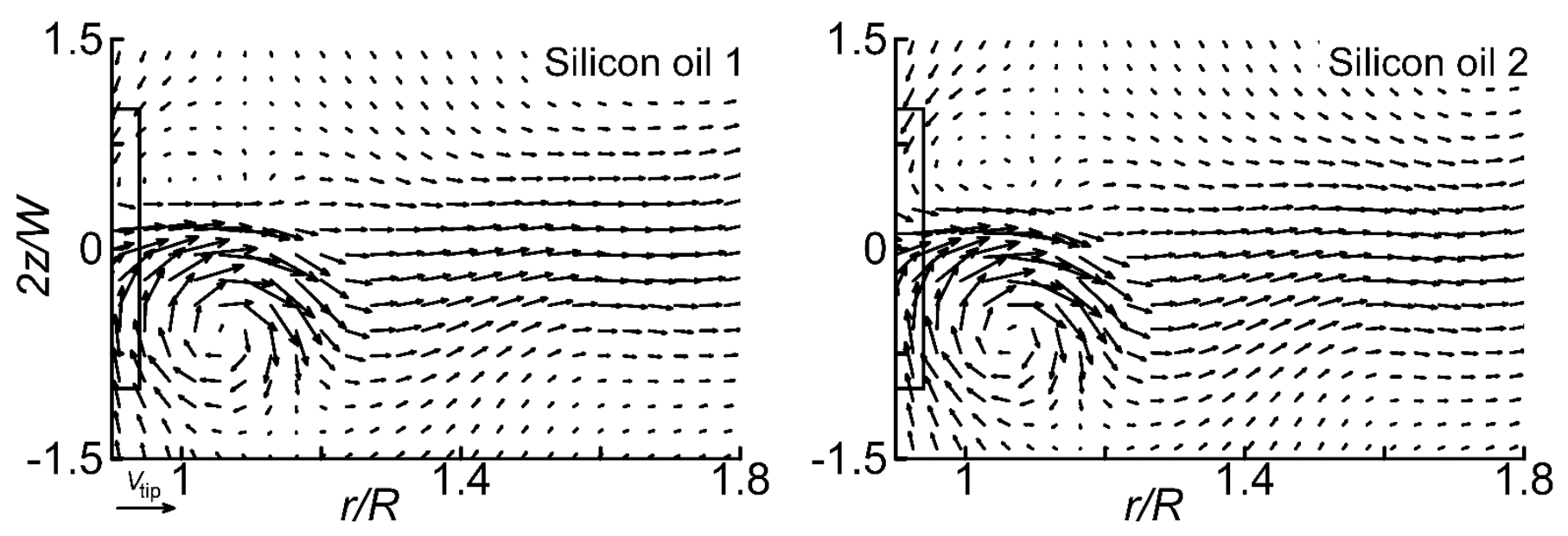

Figure 8. Phase-resolved average velocity fields at $\theta=20^{\circ}$ and $\operatorname{Re}=1,310$. Left: Silicon oil 1, SPIV data.

Right: Silicon oil 2, 2-D PIV data. 
Figure 9
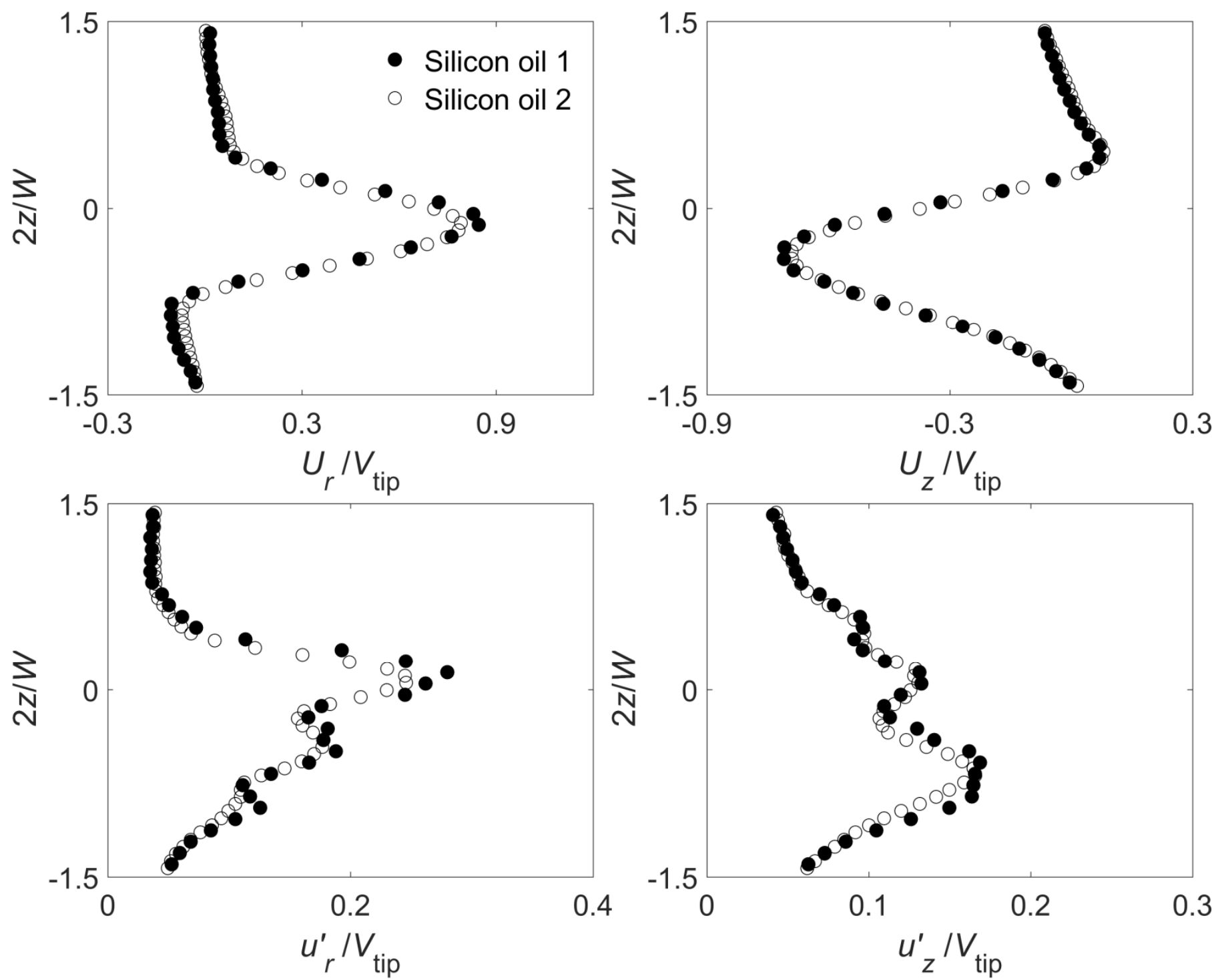

Figure 9. Comparison between results with two different liquids at the same Reynolds number $(\operatorname{Re}=1,310)$. Top row: average velocities; bottom row: velocity fluctuation levels. $r / R=1.13, \theta=20^{\circ}$. 

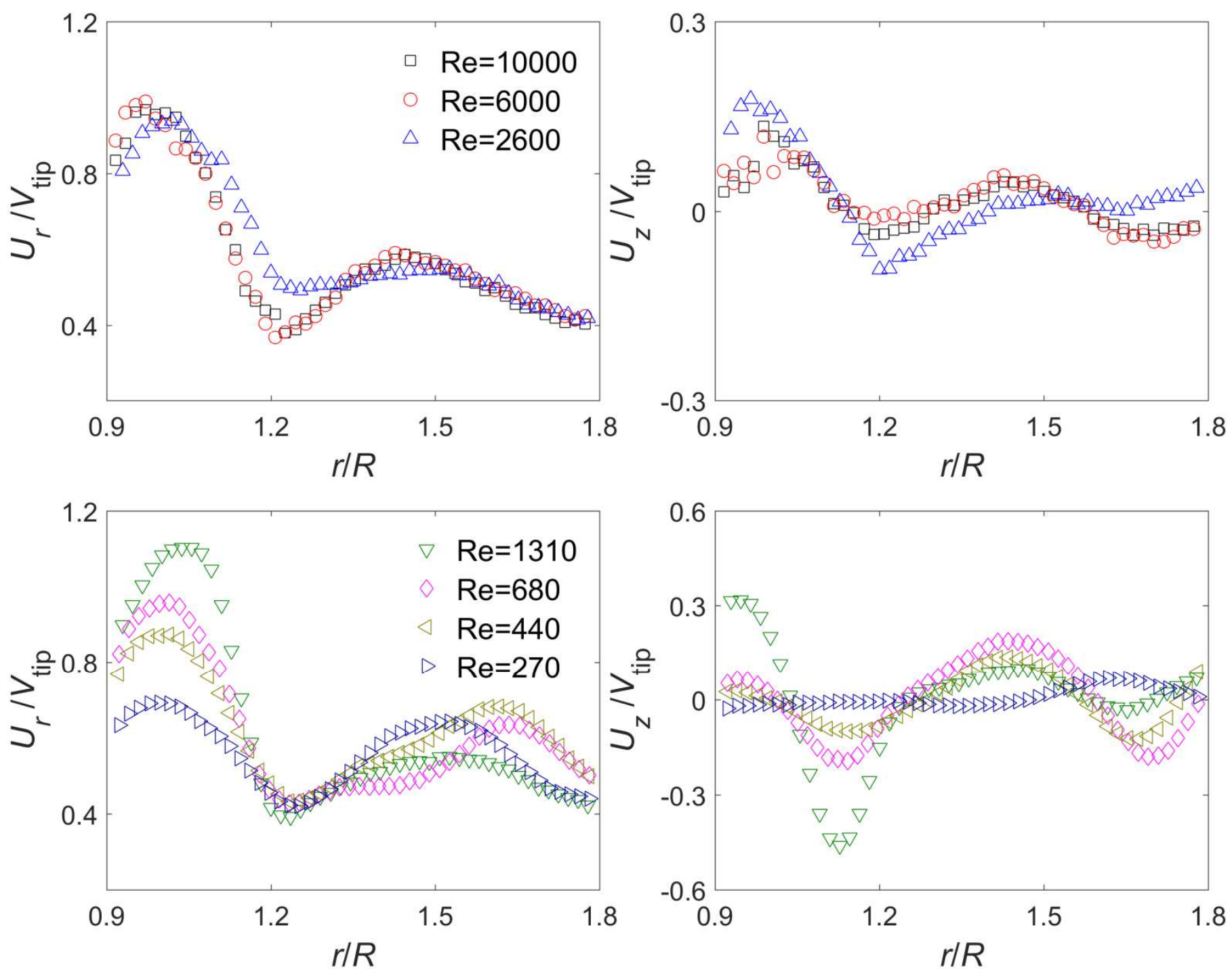

Figure 10. Radial profiles at $2 z / W=0$ for $\theta=20^{\circ}$ of average radial velocity (left) and average axial velocity (right) for a wide range of Reynolds numbers as indicated. SPIV results. 
Figure 11

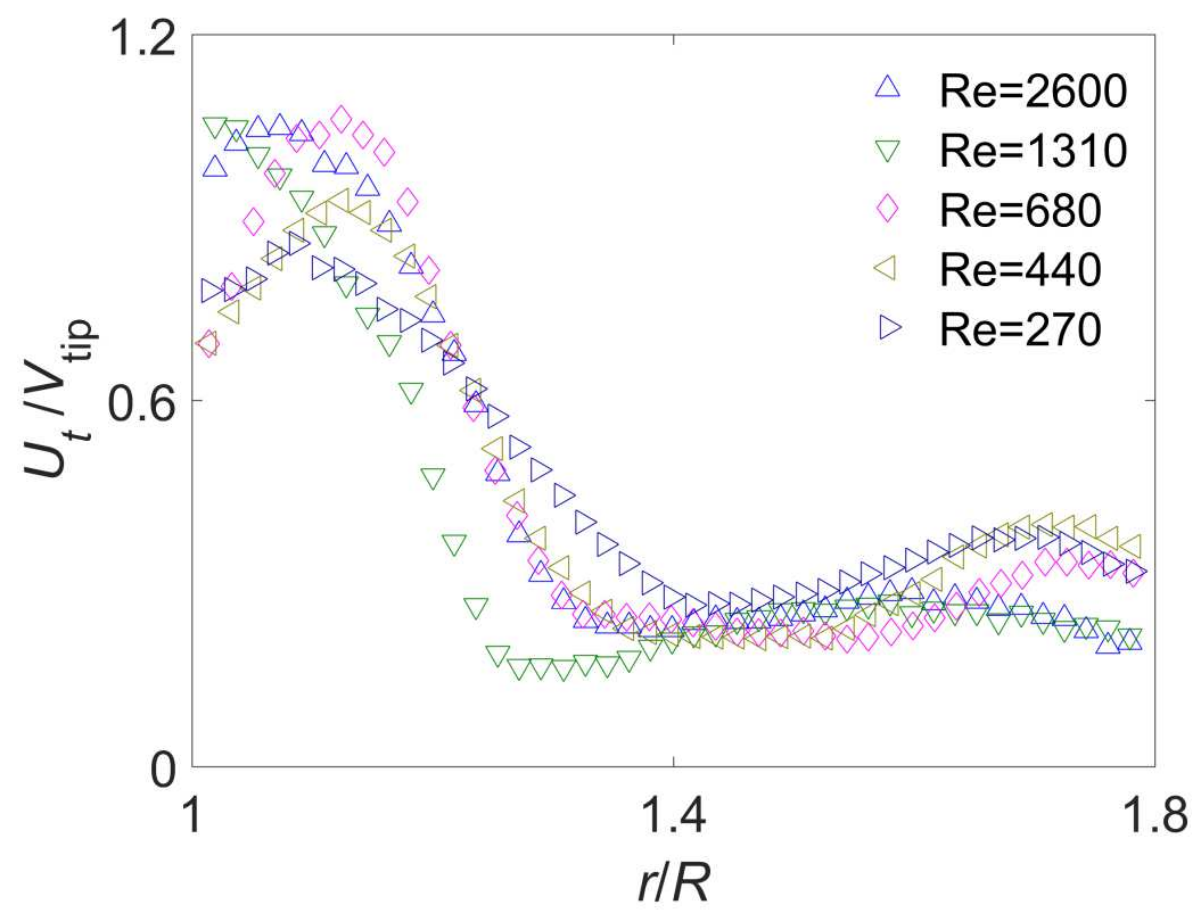

Figure 11. Radial profiles at $2 z / W=0$ for $\theta=20^{\circ}$ of average tangential velocity for a range of Reynolds numbers as indicated. SPIV results. 
Figure 12

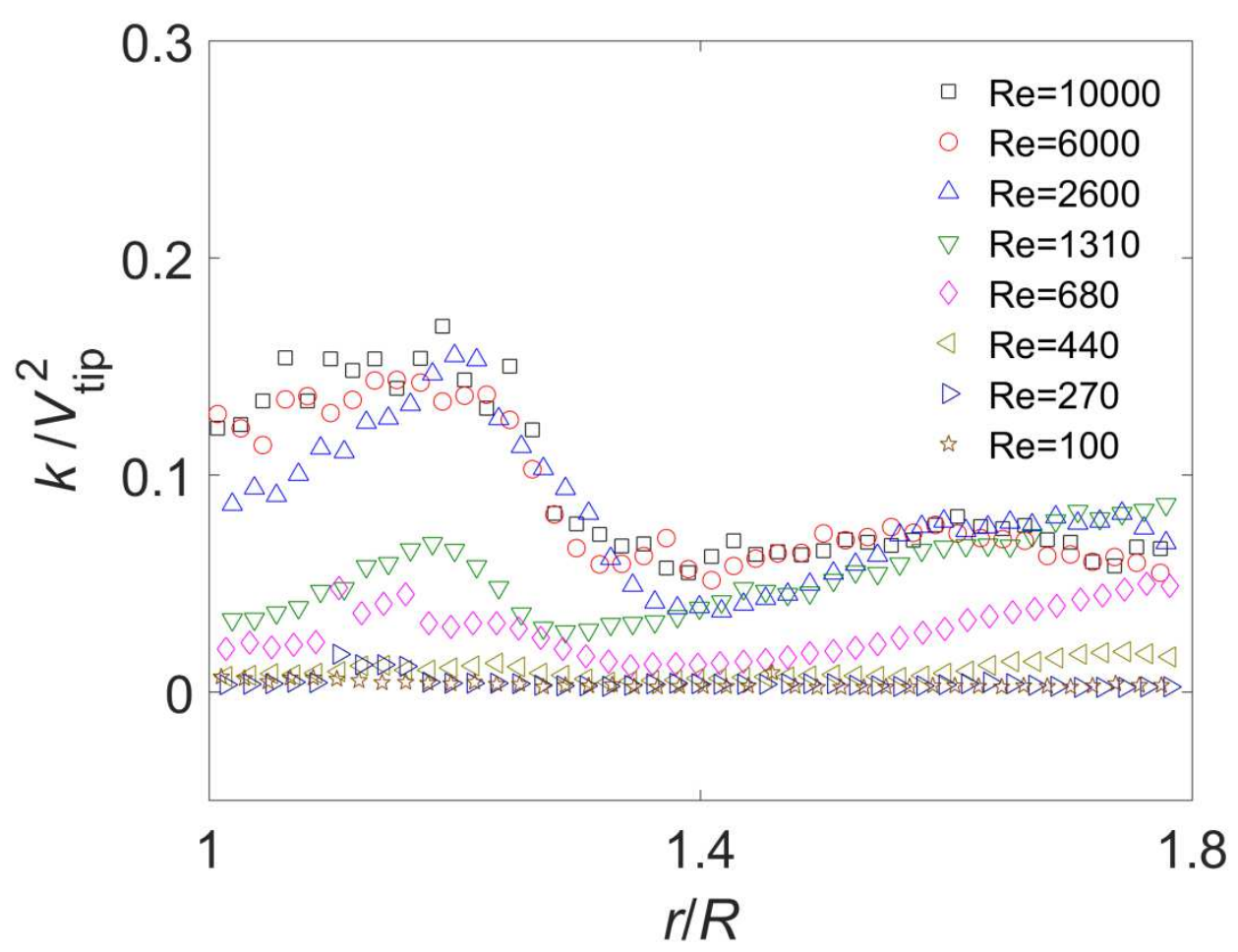

Figure 12. Radial profiles of turbulent kinetic energy at $2 z / W=0$, for $\theta=20^{\circ}$, with varying Reynolds numbers as indicated. SPIV results. 

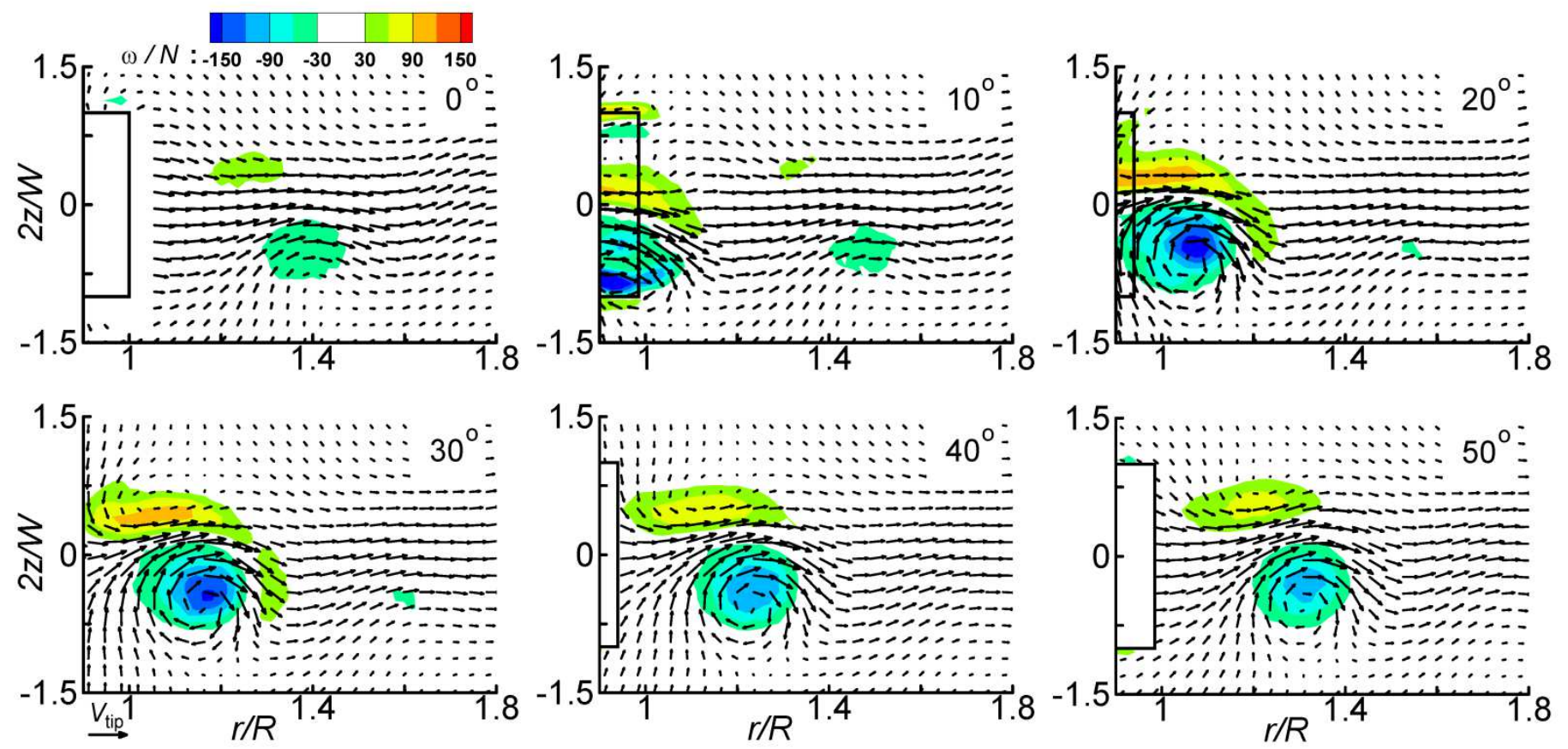

Figure 13. Phase-resolved average flow fields at $R e=1,310$ reconstructed by SPIV. The vorticity component normal to the field of view of the average flow $(\omega)$ has scaled with the angular velocity of the impeller $N$ in rev/s. Clockwise rotation has negative vorticity, counter clockwise positive. 
Figure 14
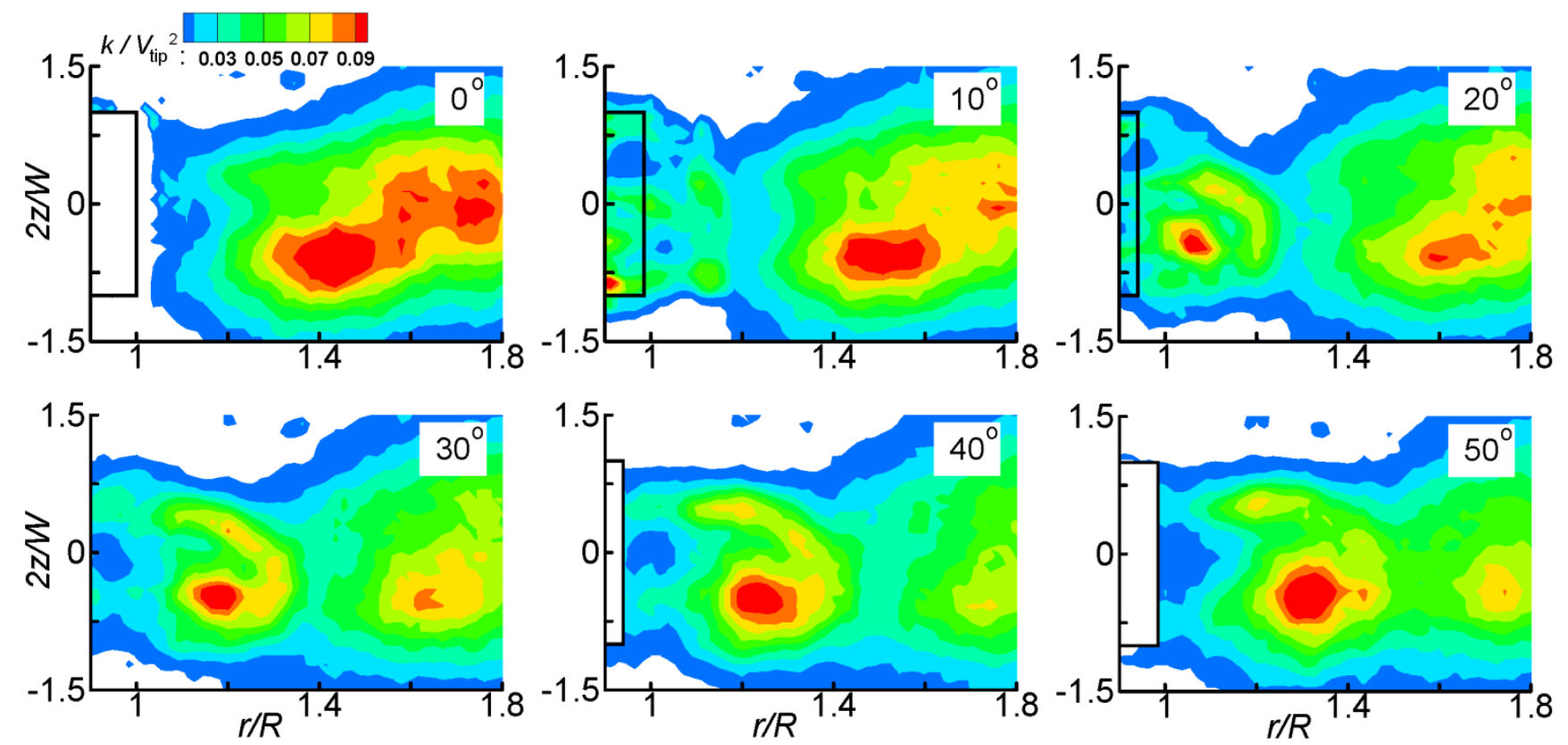

Figure 14. Turbulent kinetic energy distribution as a function of impeller angle at $R e=1,310$. SPIV results. 

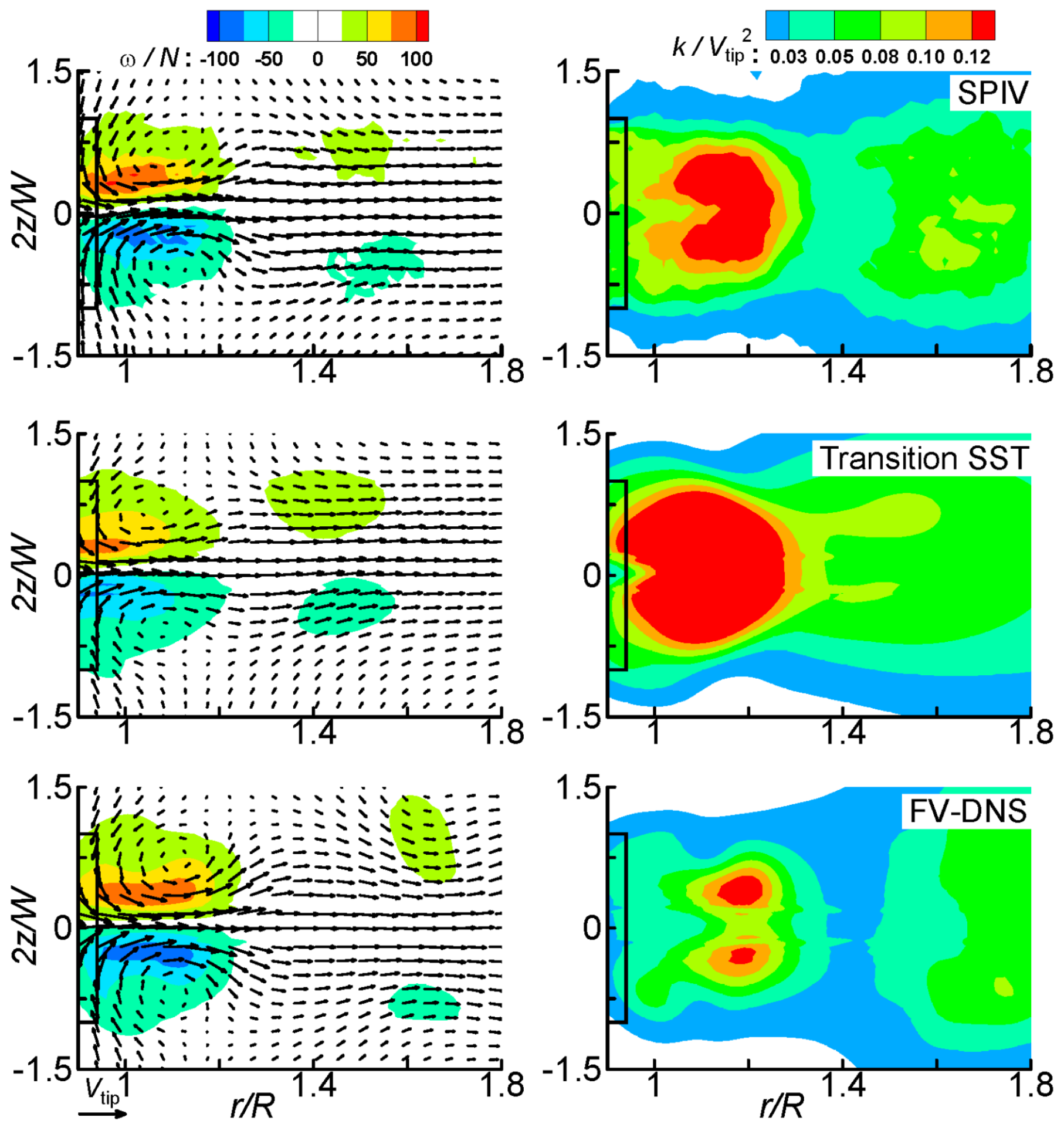

Figure 15. Phase-resolved average flow fields and turbulent kinetic energy distribution at $\theta=20^{\circ}, \operatorname{Re}=2,600 ; \mathrm{SPIV}$ versus two simulation approaches (as indicated). 

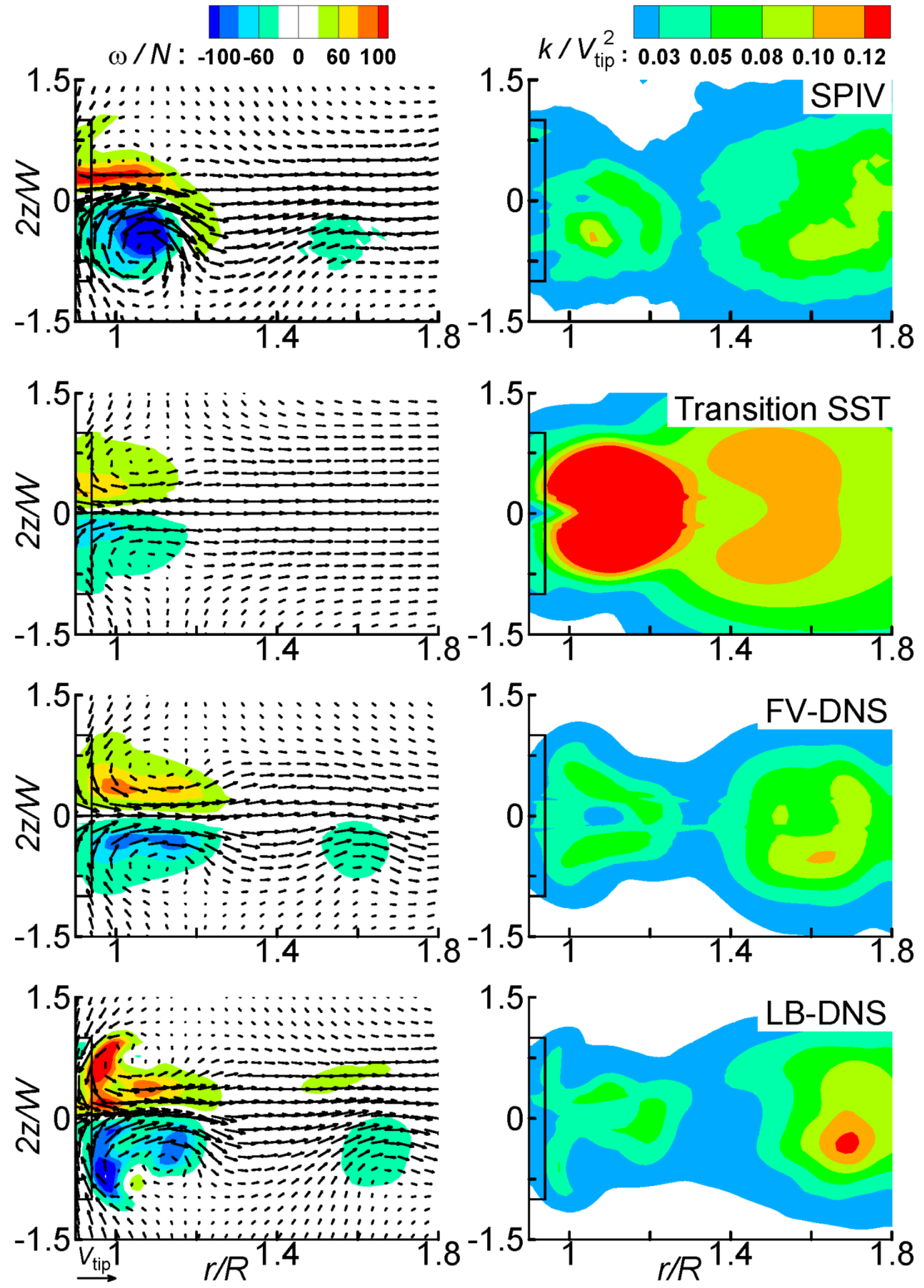

Figure 16. Phase-resolved velocity fields and turbulent kinetic energy distribution at $\theta=20^{\circ}, \operatorname{Re}=1,310$. SPIV results and three simulation approaches as indicated. 

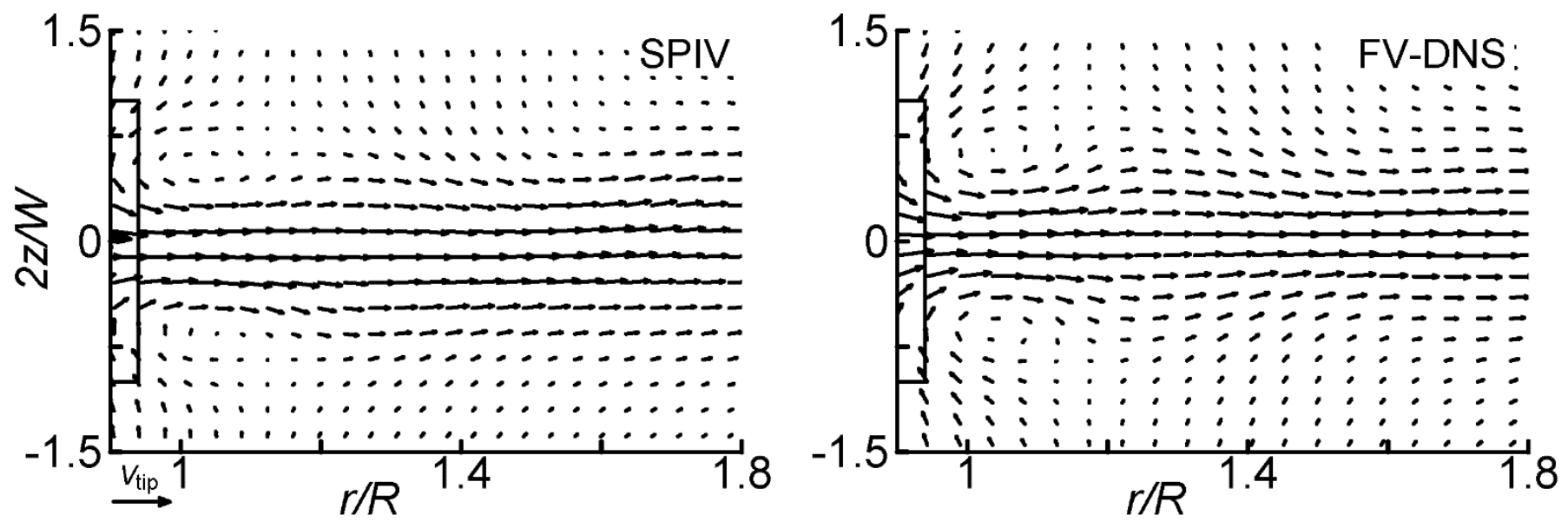

Figure 17. Phase-resolved average velocity fields at $\theta=20^{\circ}, \mathrm{Re}=270$. SPIV and FV-DNS predictions. 
Figure 18

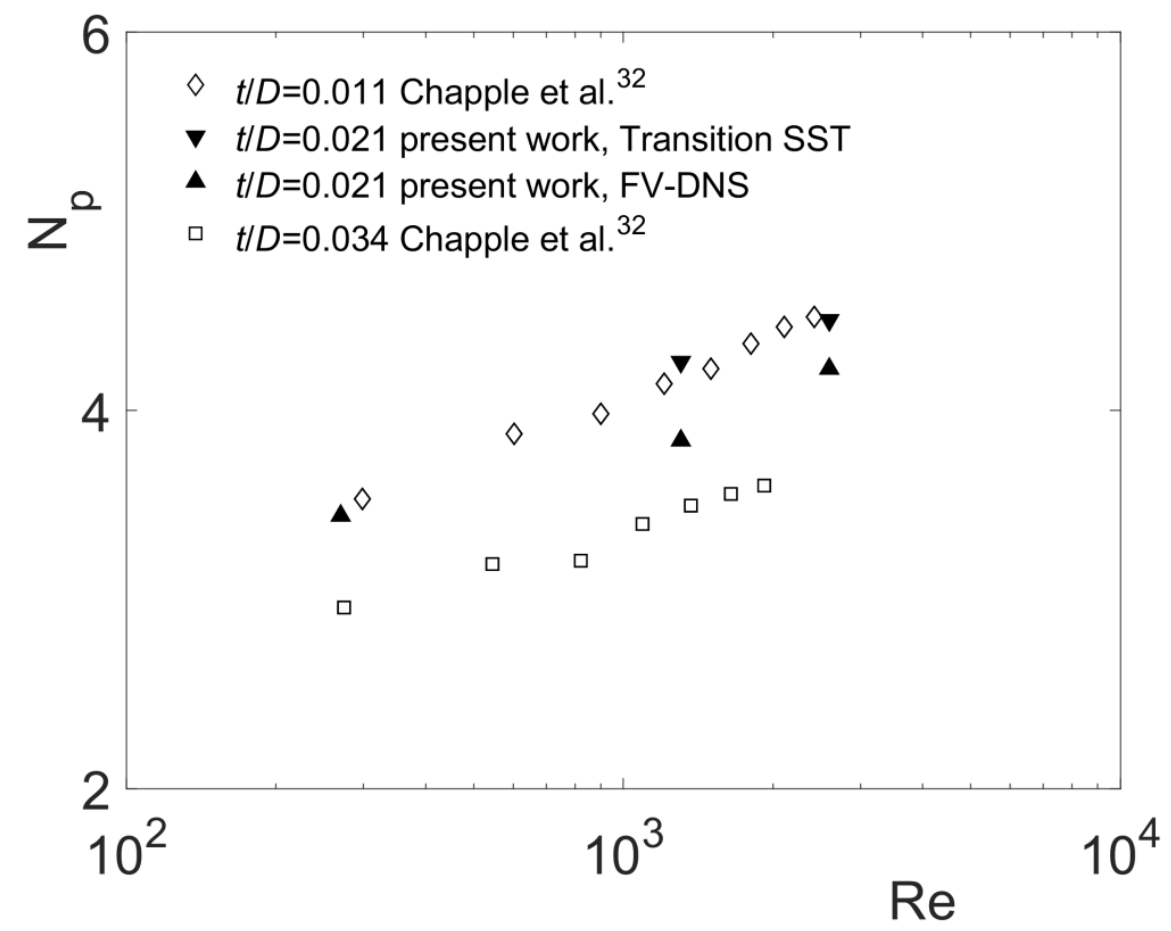

Figure 18. Power number vs. Reynolds number for a Rushton turbine in transitional flow, simulated values with two approaches and experimental data from the literature. $t / D$ is the thickness of the impeller blade relative to the impeller diameter. 